\title{
ANÁLISE DA QUALIDADE DE ENERGIA DE UM SISTEMA FOTOVOLTAICO CONECTADO A UMA REDE DE BAIXA TENSÃO
}

\author{
Márcio Zamboti Fortes \\ Doutorado em Engenharia Elétrica pela Universidade de São Paulo (USP), SP, Brasil \\ Professor Associado da Universidade Federal Fluminense (UFF), RJ, Brasil \\ mzamboti@id.uff.br \\ Vinicius Peixoto Medina \\ Mestrando em Modelagem de Sistemas de Energia na \\ Universidade Federal Fluminense (UFF), RJ, Brasil \\ viniciuspeixotomedina@hotmail.com
}

João Carlos Gonçalves Martinho

Mestrando em Engenharia Elétrica pela Universidade Federal Fluminense (UFF), RJ, Brasil

jao.martinho@gmail.com

Guilherme Vieira Cosentino

Mestrando em Metrologia e Qualidade da Inovação na Pontifícia Universidade Católica do

Rio de Janeiro (PUC-Rio), RJ, Brasil

guilhermevieira05@hotmail.com

\section{RESUMO}

A energia fotovoltaica tornou-se uma ótima alternativa aos sistemas de geração de energia elétrica tradicionais, por ser uma fonte de energia limpa e renovável. Nos últimos anos, a geração fotovoltaica para uso residencial ganhou destaque, principalmente devido aos altos custos de energia elétrica. O Brasil é um dos países com o maior custo de energia elétrica do mundo, o que motiva o uso dessa alternativa energética. No entanto, os sistemas fotovoltaicos precisam ser avaliados quanto à qualidade da energia fornecida ao consumidor. A distorção harmônica é um dos principais problemas que pode afetar a qualidade da energia. O proposto artigo consistiu na análise da qualidade de energia de um sistema fotovoltaico de baixa tensão instalado no estacionamento da Universidade Federal Fluminense (UFF). Foram realizados testes em laboratório com o objetivo de estimar a distorção harmônica total em termos de tensão e corrente e verificar se níveis recomendados foram atingidos. Os testes foram realizados com carga linear.

Palavras-chave: Geração de Energia com Sistemas Fotovoltaicos. Distorção Harmônica Total. Cargas Lineares. Inversores Fotovoltaicos. 


\title{
ANALYSIS OF POWER QUALITY ON PHOTOVOLTAIC SYSTEMS CONNECTEC TO A LOW VOLTAGE GRID
}

\begin{abstract}
The photovoltaic energy has become a great alternative to the electrical energy generation systems since it is a clean and renewable energy source. In the last years, the photovoltaic generation for residential use has been gaining emphasis, especially due to the high energy costs. Brazil is one of the countries that have one of the most electrical energy costs in the world, what motivates the use of this energy alternative. However, the photovoltaic systems need to be assessed about their delivered power quality to the consumer. Harmonic distortion is the major problem that can affect power quality. The proposed article consisted of the power quality analysis of the low voltage photovoltaic system at the Fluminense Federal University (UFF) parking, which was performed from laboratory testing with the intent to estimate the total harmonic distortion in terms of voltage and current and check if these levels were in agreement with the defined levels on the power quality standards. The tests were performed with a linear load.
\end{abstract}

Keywords: Photovoltaic Energy Generation. Total harmonic distortion. Linear Loads. Photovoltaic inverter. 


\section{INTRODUÇÃO}

A energia solar fotovoltaica é uma fonte energética renovável, ou seja, continua disponível em larga escala, mesmo com as transformações, as quais a natureza esteja sujeita a passar. A radiação solar é fundamental para a geração deste tipo de energia. O Brasil por possuir um clima tropical, apresenta um bom nível de radiação solar durante o ano, favorecendo bastante a utilização desta matriz energética. Além dos fatores citados, essa fonte de energia é limpa, não causando nenhum dano relativo à poluição ambiental no processo de geração. A capacidade instalada no Brasil ainda é inferior a países com menor incidência de radiação solar, casos de Alemanha e Espanha (PEREIRA et al., 2006). Isto representa um desafio a ser superado na política energética brasileira e estudos focados na quantificação do potencial desta fonte de energia estão apresentados em diversas pesquisas como em Magalhães et al. (2020) e Campos et al. (2020).

Apesar das metas a serem alcançadas, o parque de geração fotovoltaica no Brasil se mostra em crescente evolução, que pode ser observada se comparados os dados do Balanço Energético Nacional de 2015 (BEN 2015 - ano base de 2014), que apresentou uma capacidade instalada de geração solar fotovoltaica de $15 \mathrm{MW}$ (EMPRESA DE PESQUISA ENERGÉTICA (BRASIL), 2015), com o BEN 2018 (ano base de 2017), onde a capacidade instalada resultante foi de 935 MW (EMPRESA DE PESQUISA ENERGÉTICA (BRASIL), 2018), montante 62 vezes superior ao apresentado para o ano de 2014.

Uma política econômica estimulante à geração de fontes renováveis é essencial para alavancar a estruturação da energia fotovoltaica como política energética, e não somente, como alternativa energética. Alguns avanços no setor econômico, com relação à energia fotovoltaica, foram alcançados nos últimos anos. Financiamento, fomento econômico para as empresas nacionais e suporte para utilização do valor mínimo de consumo da eletricidade solar, são alguns destes avanços. Uma abordagem econômica da instalação de projetos fotovoltaicos residenciais está destacada em Souza et al. (2019) e um estudo especifico de perdas nas instalações fotovoltaicas em Souza et al. (2018).

No campo de políticas regulatórias, as resoluções normativas da ANEEL (Agência Nacional de Energia Elétrica) no 481 (AGÊNCIA NACIONAL DE ENERGIA ELÉTRICA, 2012a) e no 482 (AGÊNCIA NACIONAL DE ENERGIA ELÉTRICA, 2012b) tiveram bastante relevância. Estas tratam basicamente de descontos na Tarifa de Uso dos Sistemas Elétricos de Distribuição 
(TUSD) e Tarifa de Uso dos Sistemas Elétricos de Transmissão (TUST), com o intuito de estimular a geração solar em alta capacidade e o sistema de compensação para mini e microgeradores, para fomentar a geração distribuída (GD).

Os sistemas descentralizados, conectados próximos à rede de distribuição e, como consequência, próximos ao consumidor, são definidos como unidades de GD. O termo GD, nada mais é do que o significado de uma geração mais próxima do consumidor, ou seja, uma menor distância entre sistemas de geração e distribuição. A GD apresenta vantagens como redução das perdas de transmissão e dos investimentos nos sistemas de distribuição, quando comparada à geração centralizada tradicional. O sistema fotovoltaico é composto pela ligação entre várias placas fotovoltaicas que convertem a energia luminosa em energia elétrica (JURINIC, 2020).

A energia elétrica produzida em corrente contínua é convertida para corrente alternada, com o uso de um dispositivo chamado inversor. Após esta conversão, a energia passa por um filtro que reduz o nível de harmônicos de tensão e corrente, sendo assim transferida para um medidor conectado na rede elétrica, no caso de sistemas on grid.

A rede elétrica analisada foi um sistema de baixa tensão. O principal parâmetro para avaliação da qualidade de energia é o documento técnico do PRODIST (Procedimentos de Distribuição de Energia Elétrica no Sistema Elétrico Nacional) módulo 8 (AGÊNCIA NACIONAL DE ENERGIA ELÉTRICA, [2018]). Dentro dos parâmetros da qualidade de energia, o objetivo foi avaliar a distorção harmônica de tensão, tendo o próprio modulo 8 do PRODIST como referência, e a distorção harmônica de corrente, tendo como base as normas IEEE 519 (INSTITUTE OF ELECTRICAL AND ELECTRONICS ENGINEERS, 1992) e IEEE 1547 (INSTITUTE OF ELECTRICAL AND ELECTRONICS ENGINEERS, 2003).

O objetivo deste trabalho foi mostrar o estudo da qualidade de energia de um sistema fotovoltaico on grid, ou seja, conectado a uma rede elétrica.

\section{REFERENCIAL TEÓRICO}

Existem diversos estudos sobre o impacto dos sistemas fotovoltaicos conectados à rede de distribuição de energia elétrica no aspecto qualidade de energia como apresentado em Afonso et al. (2018) e Bouchakour et al. (2017). 
A figura 1 representa o diagrama esquemático para um sistema fotovoltaico on grid, em uma rede elétrica de baixa tensão similar ao estudado em LEITE et al. (2018).

Figura 1 - Diagrama esquemático de um sistema fotovoltaico on grid.

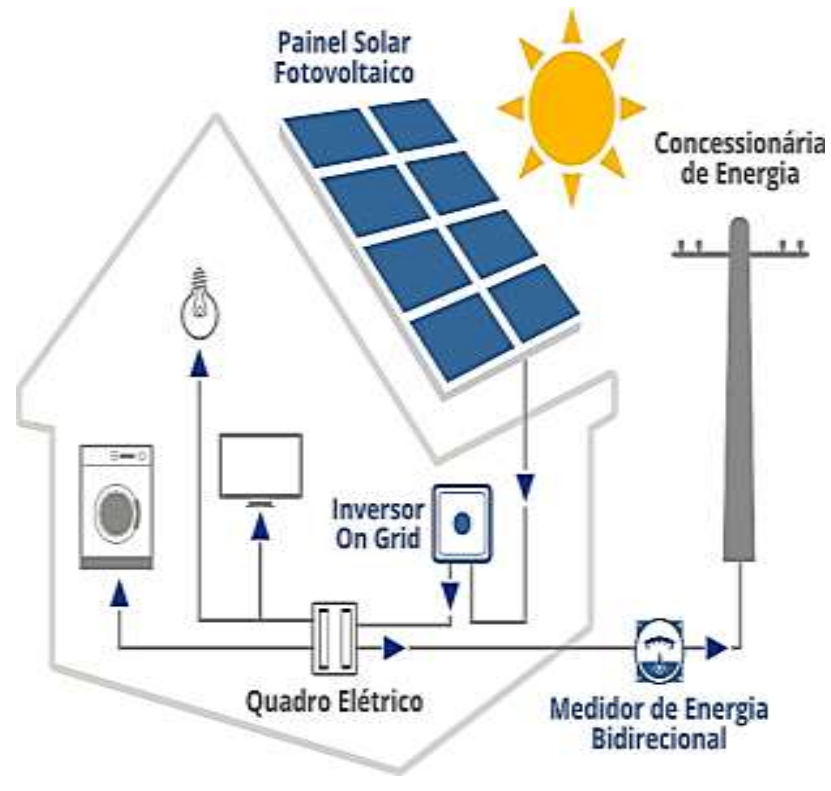

Fonte: Aline (2018).

No Brasil, a referência para os conceitos de qualidade de energia é aquela encontrada no documento técnico PRODIST módulo 8 (AGÊNCIA NACIONAL DE ENERGIA ELÉTRICA, [2018]). Alguns parâmetros de qualidade de energia serão retratados neste trabalho, como a distorção harmônica e o fator de potência, pois foram as variáveis de qualidade de energia avaliadas no experimento realizado, o qual será descrito no item 3 deste artigo.

Os subtópicos a seguir promovem a explanação destes parâmetros.

\subsection{Conceito de Distorção Harmônica}

Distorção harmônica é toda alteração no formato da onda de tensões e correntes em relação ao formato de onda senoidal da frequência fundamental. Estudos sobre distorção harmônicas e seus impactos nos sistemas de energia ou equipamentos estão reportados em diferentes pesquisas como em Vidaurre e Fortes (2020) e Silva et al. (2020). A 
distorção harmônica pode ser dividida em: distorção harmônica de tensão e de corrente. Ambas as distorções serão abordadas nesse trabalho.

\subsection{Distorção Harmônica de Tensão}

A distorção harmônica de tensão se divide em várias terminologias, conforme pode ser conferido no quadro 1.

Quadro 1 - Terminologia para Distorção Harmônica de Tensão.

\begin{tabular}{|c|c|}
\hline Grandeza & Acrônimo \\
\hline Distorção harmônica individual de tensão de ordem $\mathrm{h}$ & $\mathrm{DTT}_{\mathrm{h}} \%$ \\
\hline Distorção harmônica total de tensão & DTT\% \\
\hline $\begin{array}{l}\text { Distorção harmônica total de tensão para as componentes } \\
\text { pares não múltiplas de } 3\end{array}$ & $\mathrm{DTT}_{\mathrm{p}} \%$ \\
\hline $\begin{array}{l}\text { Distorção harmônica total de tensão para as componentes } \\
\text { ímpares não múltiplas de } 3\end{array}$ & DTT,\% \\
\hline $\begin{array}{l}\text { Distorção harmônica total de tensão para as componentes } \\
\text { múltiplas de } 3\end{array}$ & $\mathrm{DTT}_{3} \%$ \\
\hline Tensão harmônica de ordem $\mathrm{h}$ & $V_{h}$ \\
\hline Ordem harmônica máxima & $\mathrm{h}_{\max }$ \\
\hline Ordem harmônica mínima & $h_{\min }$ \\
\hline Tensão fundamental medida & $V_{1}$ \\
\hline $\begin{array}{l}\text { Valor indicador DTT\% que foi superado em apenas 5\% das } \\
1008 \text { leituras válidas }\end{array}$ & DTT95\% \\
\hline $\begin{array}{l}\text { Valor indicador } \mathrm{DTT}_{\mathrm{p}} \% \text { que foi superado em apenas } 5 \% \text { das } \\
1008 \text { leituras válidas }\end{array}$ & $\mathrm{DTT}_{\mathrm{p}} 95 \%$ \\
\hline $\begin{array}{l}\text { Valor indicador } \mathrm{DTT}_{1} \% \text { que foi superado em apenas } 5 \% \text { das } \\
1008 \text { leituras válidas }\end{array}$ & $\mathrm{DTT}_{1} 95 \%$ \\
\hline $\begin{array}{l}\text { Valor indicador } \mathrm{DTT}_{3} \% \text { que foi superado em apenas } 5 \% \text { das } \\
1008 \text { leituras válidas }\end{array}$ & $\mathrm{DTT}_{3} 95 \%$ \\
\hline
\end{tabular}

Fonte: Agência Nacional De Energia Elétrica ([2018]).

Este artigo irá abordar apenas a definição da terminologia DTT (Distorção harmônica total de tensão). Esta pode ser definida a partir da Equação (1).

$$
\operatorname{DTT}(v)=\frac{\sum_{h=2}^{h m a ́ x} V_{h}{ }^{2}}{V_{1}}
$$

Fonte: Autor (2020). 
Onde $h$ são as componentes de ordem harmônicas, variando da ordem 2 até a ordem $h m a ́ x ; ~ V_{h}$ é a tensão medida na ordem harmônica $h$; hmáx é a ordem harmônica máxima e $V_{1}$ é a tensão medida na ordem fundamental.

\subsection{Distorção Harmônica de Corrente}

Em (AGÊNCIA NACIONAL DE ENERGIA ELÉTRICA, [2018]) não há uma definição formal sobre distorção harmônica de corrente. Com base na norma IEEE 1547 (INSTITUTE OF ELECTRICAL AND ELECTRONICS ENGINEERS, 2003), esta distorção pode ser definida de acordo com a Equação (2).

$$
\operatorname{DTT}(i)=\frac{\sum_{h=2}^{h m x^{x}} I_{h}{ }^{2}}{I_{1}}
$$

Fonte: Autor (2020).

Onde $h$ são as componentes harmônicas, variando da ordem 2 até a ordem $h_{\text {máx; }} I_{h}$ é a corrente medida na ordem harmônica $h ; h_{\text {máx }}$ é a ordem harmônica máxima e $l_{1}$ é a corrente medida na ordem fundamental.

\subsection{Fator de Potência}

O fator de potência define a razão entre a potência ativa e a potência aparente. Pode ser calculado, segundo a Agência Nacional De Energia Elétrica ([2018]), de acordo com a Equação (3).

$$
F P=\frac{P}{S}=\frac{P}{\sqrt{P^{2}+S^{2}}}
$$

Fonte: Autor (2020).

Onde FP é o fator de potência adimensional; S é a potência aparente em VoltAmpere; $\mathrm{P}$ é a potência elétrica ativa em Watts e $\mathrm{S}$ é a potência elétrica reativa em VoltAmpere-Reativo. 
O fator de potência é de suma importância em um sistema de potência, pois este define a eficiência do aproveitamento da potência aparente fornecida ao sistema, ou seja, qual percentual daquela potência foi realmente aproveitado pelo sistema (GOFFERJÉ, 2019).

Em (AGÊNCIA NACIONAL DE ENERGIA ELÉTRICA, [2018]) fazem-se algumas considerações sobre o fator de potência, em termos de qualidade de energia. Dentre as quais, podem ser citadas: o controle do fator de potência deve ser realizado a partir de medições permanentes e obrigatórias para unidades consumidoras de média e alta tensão e para as conexões entre as distribuidoras. Para instalações de baixa tensão, as medições são facultativas. No que se refere aos valores de referência do fator de potência, este pode ser definido de acordo com a unidade que acessa o sistema de potência, podendo esta ser uma unidade consumidora ou uma central geradora.

Em uma unidade consumidora ou em um ponto de conexão com a distribuidora, no qual a tensão é inferior a 230 kV, o fator de potência deve estar entre 0,92 e 1,00, podendo ser indutivo ou capacitivo. Já para unidades consumidoras que acessam à rede básica, o fator de potência deve seguir os padrões determinados nos procedimentos de rede. No que se refere às centrais geradoras, o fator de potência também deve seguir os procedimentos de rede. A potência reativa quando em excesso, pode ocasionar problemas chamados de excedentes reativos, os quais além de afetar os níveis de distorção harmônica, afetam também os níveis aceitáveis de tensão num sistema de potência, provocando desequilíbrio e flutuação de tensão, além de uma possível inversão no sentido do fluxo de potência, podendo causar problemas chamados de ilhamento em sistemas de potência (RIBEIRO JR., 2015).

\section{MATERIAIS E MÉTODOS}

Este estudo consiste na análise de alguns parâmetros da qualidade de energia de um sistema fotovoltaico constituído de um painel solar, um inversor, um transformador e dois conjuntos de cargas lineares conforme a figura 2. 
Figura 2 - Esquema experimental.

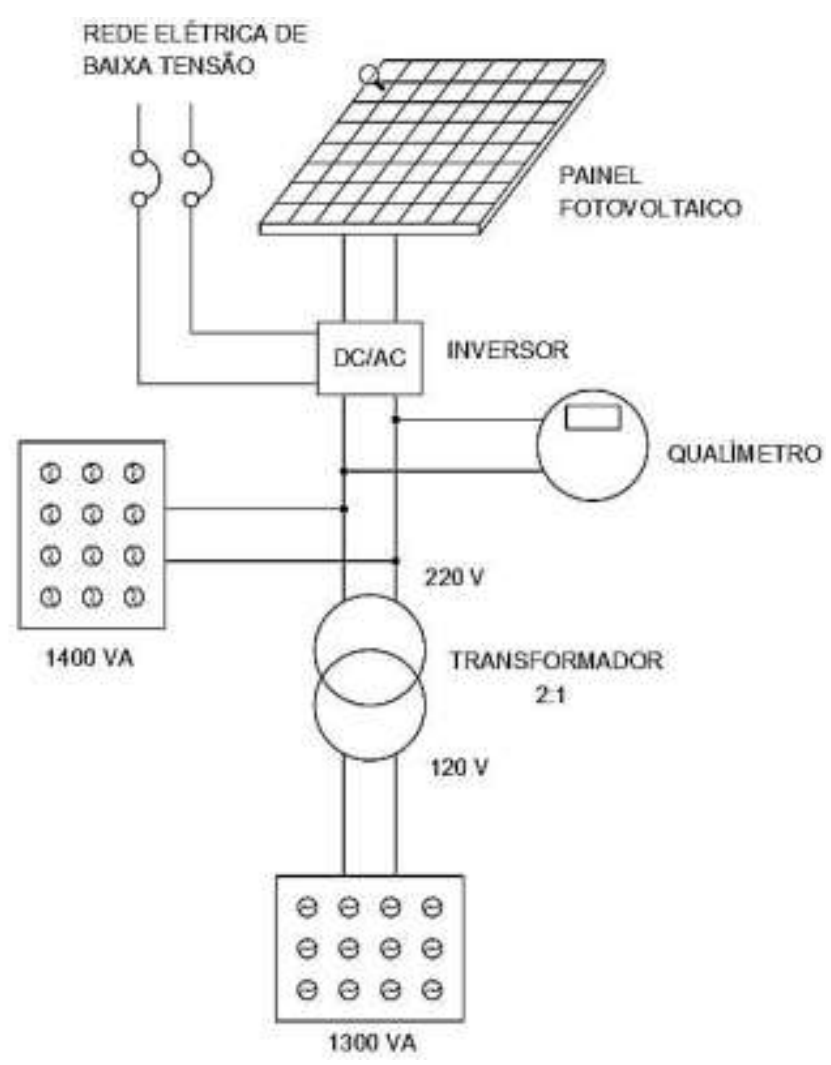

Fonte: Autor (2020).

A simulação da geração solar foi realizada a partir do painel solar instalado no estacionamento da Universidade Federal Fluminense (UFF) no campus da Praia Vermelha. Foi utilizado um software online SunData para gerar informações necessárias quanto à radiação solar no painel estudado.

Em relação aos parâmetros da qualidade de energia, foi possível avaliar os harmônicos de tensão e corrente nos terminais de saída do inversor e verificar para quais cenários de carga os harmônicos de tensão e corrente seriam mais relevantes em relação às características nominais. Além disso, foi possível analisar a influência de não linearidade por parte da carga. A carga utilizada foi puramente resistiva, dimensionada somente com lâmpadas incandescentes, totalizando 2700VA de potência. O transformador foi utilizado devido a um dos grupos de lâmpadas utilizado como carga ter especificação de 120/127 V, enquanto o inversor fornecia uma tensão de saída de $220 \mathrm{~V}$.

O sistema de placas fotovoltaicas foi organizado de modo que as duas strings de 9 placas fossem ligadas em paralelo, com uma tensão de $300 \mathrm{~V}$ e uma corrente de até $18 \mathrm{~A}$, 
em detrimento da carga utilizada. A parte experimental foi realizada no dia 24 de junho de 2019 , entre as $12 \mathrm{~h}$ e às $15 \mathrm{~h}$, sendo dividida em dois conjuntos de ensaios: Alimentação pela rede e alimentação pelo inversor conectado à rede. Na alimentação pela rede, utilizou-se a conexão sem o inversor, a carga foi alimentada somente pela rede elétrica, no segundo ensaio utilizou-se o esquema da figura 2 para alimentação com variações de carga de $15 \%$ em relação à nominal, ou seja, 100\%, 85\%, 60\%, 45\%, 30\% e 15\%.

O sistema fotovoltaico era inicialmente isolado (não conectado à rede), porém para a realização dos ensaios, ao conectá-lo com um inversor ligado à rede elétrica, este sistema passou a ser conectado à rede elétrica de baixa tensão.

O método de cálculo utilizado foi realizado a partir de um gerenciador de sinais. Em todas as situações analisadas, o fator de potência era unitário.

O painel foi composto por 18 módulos constituídos de 60 células, com potência de 250 Wp cada. Nos quadros II e III são apresentadas as especificações técnicas elétricas e mecânicas para o padrão STC (Standard Test Conditions), fornecidas pelo respectivo fabricante das células.

Quadro 2 - Características elétricas para um padrão STC da célula fotovoltaica.

\begin{tabular}{|l|l|}
\hline Número de Células & 60 \\
\hline Potência de Saída & $250 \mathrm{Wp}$ \\
\hline Eficiência & $15,4 \%$ \\
\hline Tensão em máxima potência & $29,8 \mathrm{~V}$ \\
\hline Corrente em máxima potência & $8,39 \mathrm{~A}$ \\
\hline
\end{tabular}

Fonte: Autor (2020).

Quadro 3 - Características mecânicas para um padrão STC da célula fotovoltaica.

\begin{tabular}{|l|l|}
\hline Tipo de Célula & Silício Policristalino \\
\hline Dimensões & $164 \times 990 \times 35 \mathrm{~mm}$ \\
\hline Peso & $18,5 \mathrm{~kg}$ \\
\hline Grau de Proteção & $\mathrm{IP65}$ \\
\hline Comprimento dos cabos de conexão & $1000 \mathrm{~mm}$ \\
\hline Seção dos cabos de conexão & $4 \mathrm{~mm}^{2}$ \\
\hline Tipo de Conector & $\mathrm{MC} 4$ \\
\hline
\end{tabular}

Fonte: Autor (2020). 
$\mathrm{Na}$ figura 3 ilustra-se a disposição que foi utilizada para os 18 módulos fotovoltaicos.

Figura 3 - Disposição dos módulos fotovoltaicos utilizados.

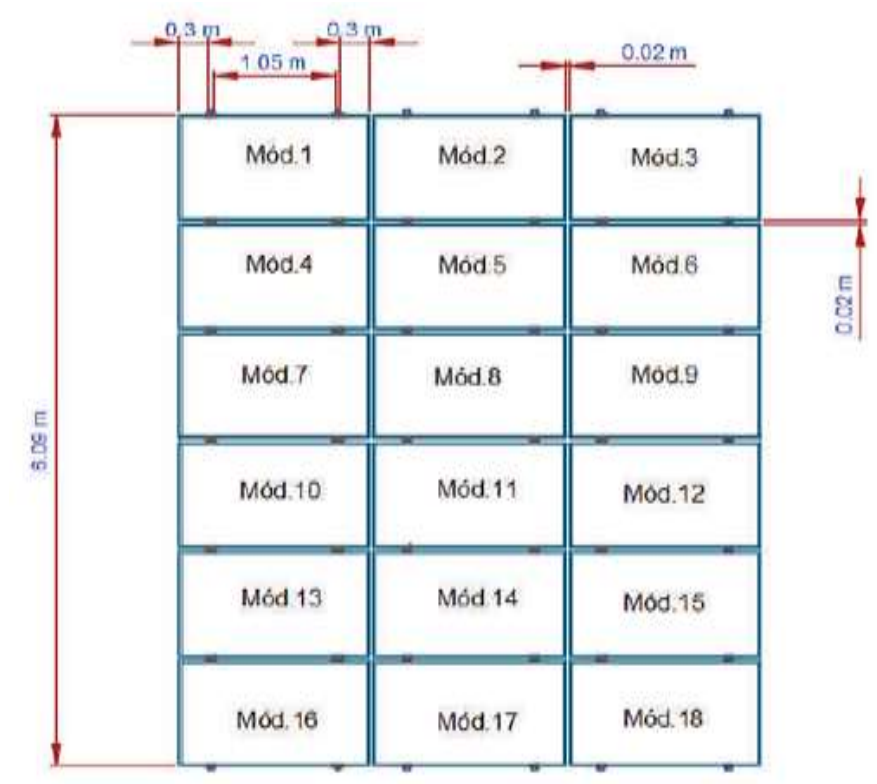

Fonte: Autor (2020).

Essa conexão possibilitou uma potência de saída de $4,5 \mathrm{kWp}$, onde o valor mínimo de operação para tensão foi de $120 \mathrm{~V}$ e o máximo, de $580 \mathrm{~V}$. O quadro 4 mostra as especificações técnicas mais importantes para o inversor utilizado, de acordo com os dados do fabricante.

Quadro 4 - Principais Especificações do Inversor.

\begin{tabular}{|l|l|}
\hline \multicolumn{2}{|c|}{ Dados de Entrada } \\
\hline Máxima Potência Fotovoltaica & $3000 \mathrm{~W}$ \\
\hline Máxima Tensão CC & $500 \mathrm{~V}$ \\
\hline Máxima Corrente CC & $15 \mathrm{~A}$ \\
\hline \multicolumn{2}{|c|}{ Dados de Saída } \\
\hline Potência CA Nominal & $3000 \mathrm{~W}$ \\
\hline Máxima Potência CA & $3000 \mathrm{~W}$ \\
\hline Máxima Corrente CA & $13,5 \mathrm{~A}$ \\
\hline Saída nominal & $60 \mathrm{~Hz} ; 220 \mathrm{Vca}$ \\
\hline THD & $<5 \%$ \\
\hline Fator de Potência & 1 \\
\hline Conexão CA & Monofásica \\
\hline
\end{tabular}

Fonte: Autor (2020). 
Foram montados dois conjuntos de lâmpadas para representarem cargas lineares conectadas ao sistema, como mostrado na figura 2.

A aquisição de dados de qualidade de energia foi realizada com o uso de um instrumento denominado qualímetro ou analisador de energia. Este equipamento grava novos dados a cada 200 milissegundos e, no modelo experimental, utilizou-se quadros de dados que apresentaram valores máximos de DTT de tensão e corrente na situação carga analisada.

\section{RESULTADOS OBTIDOS}

Esse tópico trata dos resultados obtidos para os dois conjuntos de ensaios realizados: A alimentação da carga com o qualímetro conectado diretamente à rede, sem inversor e o outro ensaio com a ligação na rede sendo feita com o uso de inversor e do sistema fotovoltaico do local. Esses resultados trataram de mostrar quais foram os valores das distorções harmônicas de corrente e de tensão, para os dois tipos de ensaios realizados. Para ambos os ensaios, foram construídas tabelas que descreviam a distribuição percentual das correntes e tensões medidas em relação à ordem fundamental, além de gráficos de colunas, para facilitar a visualização dos resultados em quadros.

\subsection{Arranjo experimental alimentado pela rede}

Pode-se verificar que o DTT de corrente apresentou o valor de $3,152 \%$ e suas respectivas contribuições harmônicas estão disponíveis no quadro 5.

Quadro 5 - Distribuição Percentual das correntes medidas em relação à ordem harmônica fundamental.

\begin{tabular}{|l|l|l|}
\hline $\mathrm{N}^{\circ}$ da ordem & $\mathrm{I}_{\text {rms }}(\mathrm{A})$ & \% fundamental \\
\hline 1 & 12,39 & 100 \\
\hline 3 & 0,12 & 1 \\
\hline 5 & 0,30 & 2,47 \\
\hline 7 & 0,11 & 0,89 \\
\hline
\end{tabular}

Fonte: Autor (2020). 
A figura 4 retrata a acomodação das informações do quadro 5, com o objetivo de representar o comportamento dos harmônicos de corrente de ordem impar em relação à fundamental.

Figura 4 - Harmônicos de corrente, sistema alimentado pela rede.

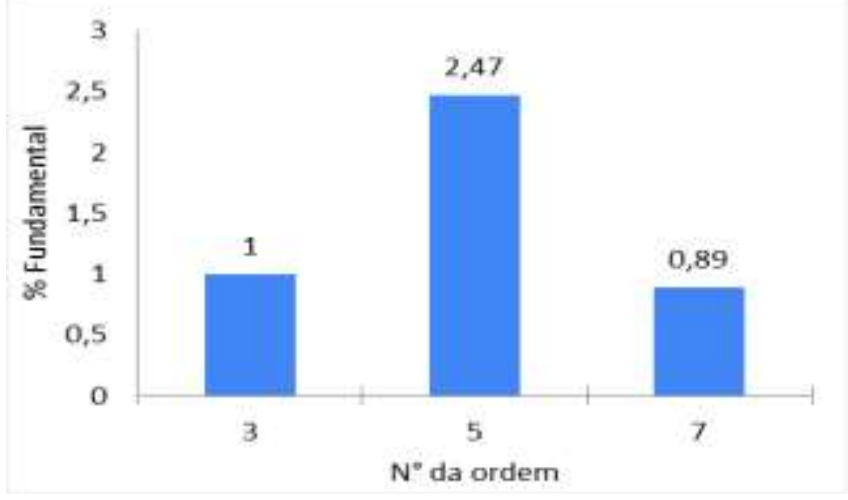

Fonte: Autor (2020).

Analisando os harmônicos de tensão, ainda na situação do sistema conectado somente à rede, pode-se averiguar o cenário expresso no quadro 6. Esta foi a representação da qualidade de energia fornecida pela distribuidora no ponto de conexão do cliente.

Quadro 6 - Harmônicos de Tensão, sistema alimentado pela rede.

\begin{tabular}{|l|l|l|}
\hline $\mathrm{N}^{\circ}$ da ordem & $\mathrm{V}_{\text {rms }}(\mathrm{V})$ & \% fundamental \\
\hline 1 & 217,91 & 100 \\
\hline 3 & 1,20 & 0,55 \\
\hline 5 & 5,14 & 2,36 \\
\hline 7 & 1,66 & 0,76 \\
\hline
\end{tabular}

Fonte: Autor (2020).

Foi possível expressar numericamente o valor do DTT de tensão como sendo de $2,542 \%$.

A figura 5 representa a organização das informações do quadro 6, com a finalidade de descrever o comportamento dos harmônicos de tensão de ordem impar em relação a fundamental. 
Figura 5 - Harmônicos de tensão, sistema alimentado pela rede.

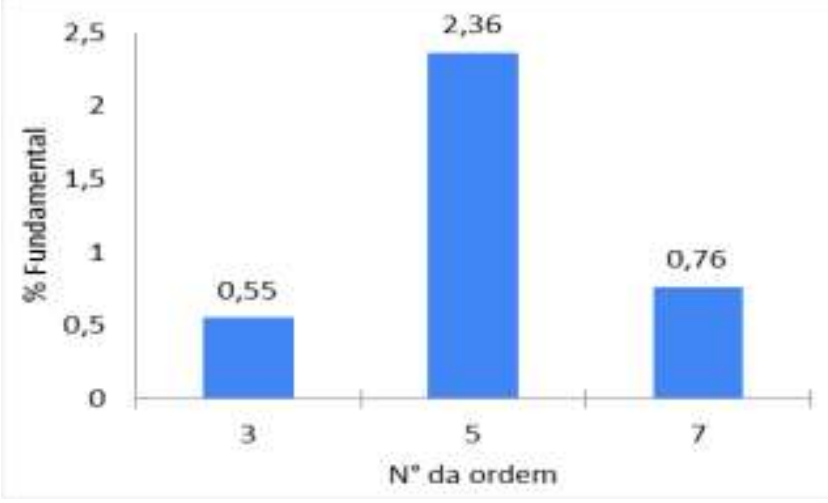

Fonte: Autor (2020).

Os valores do DTT de corrente e tensão estão dentro dos valores normalizados, segundo o Institute Of Electrical And Electronics Engineers (1992) e a Agência Nacional De Energia Elétrica ([2018]), respectivamente.

\subsection{Arranjo experimental alimentado pelo inversor}

\subsubsection{Carga nominal ou $100 \%$ da carga}

O primeiro arranjo experimental alimentado pelo inversor e conectado à rede traduz-se com situação de carga nominal. O sistema será alimentado pelo inversor e, ocorrerá avaliação da qualidade de energia disponibilizada, na saída do equipamento.

Neste primeiro cenário, foram analisados os harmônicos com uma carga de 2.700VA. Pode-se verificar que o DTT de corrente apresentou o valor de 3,48\% e suas respectivas contribuições harmônicas estão disponíveis no Quadro 7.

Quadro 7 - Harmônicos de Corrente, sistema alimentado pelo inversor, com valor máximo de carga.

\begin{tabular}{|l|l|l|}
\hline $\mathrm{N}^{\circ}$ da ordem & $\mathrm{I}_{\text {rms }}(\mathrm{A})$ & \% fundamental \\
\hline 1 & 12,37 & 100 \\
\hline 3 & 0,12 & 1 \\
\hline 5 & 0,33 & 2,67 \\
\hline 7 & 0,10 & 0,85 \\
\hline
\end{tabular}

Fonte: Autor (2020). 
A figura 6 reflete a disposição das informações no quadro 7, com a finalidade de estruturar o comportamento dos harmônicos de corrente de ordem impar em relação à fundamental.

Figura 6 - Harmônicos de corrente, sistema alimentado pelo inversor, com carga máxima.

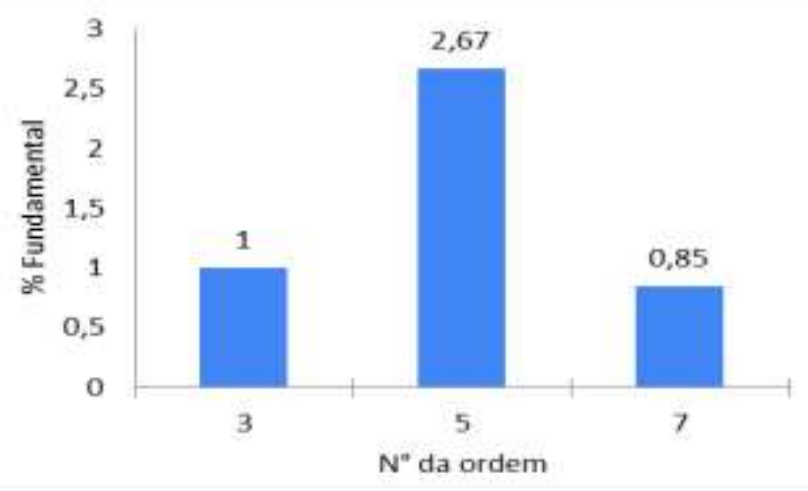

Fonte: Autor (2020).

Analisando os harmônicos de tensão, na situação do sistema alimentado pelo inversor conectado à rede, com carga nominal, pode-se averiguar o cenário expresso no quadro 8. Representa-se numericamente o valor do DTT de tensão como sendo de $2,85 \%$.

Quadro 8 - Harmônicos de Tensão, sistema alimentado pelo inversor, com valor máximo de carga.

\begin{tabular}{|l|l|l|}
\hline$N^{\circ}$ da ordem & $V_{\text {rms }}(A)$ & $\begin{array}{l}\% \\
\text { fundamental }\end{array}$ \\
\hline 1 & 218,52 & 100 \\
\hline 3 & 1,34 & 0,61 \\
\hline 5 & 5,89 & 2,70 \\
\hline 7 & 1,55 & 0,71 \\
\hline
\end{tabular}

Fonte: Autor (2020).

A figura 7 representa as informações do quadro 8, de maneira gráfica, com a intenção de representar o comportamento dos harmônicos de tensão de ordem impar em relação à fundamental. 
Figura 7 - Harmônicos de corrente, sistema alimentado pelo inversor, com carga máxima.

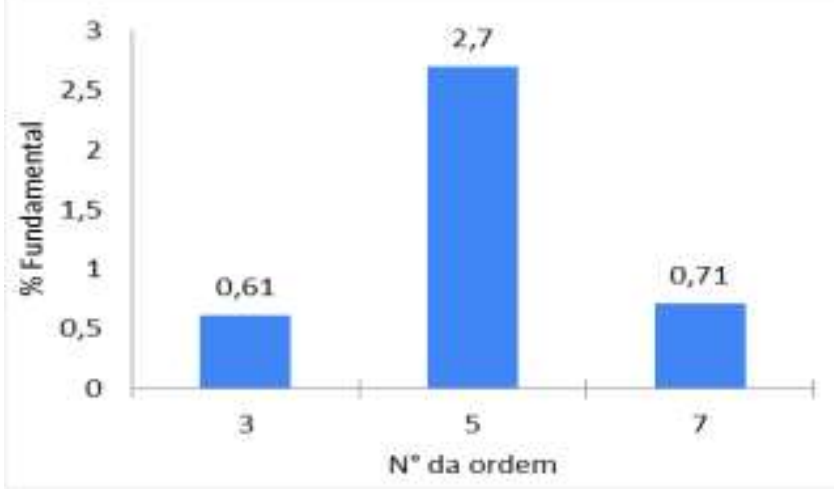

Fonte: Autor (2020).

Os valores do DTT de corrente e tensão estão dentro dos valores normalizados, segundo o Institute Of Electrical And Electronics Engineers (1992) e a Agência Nacional De Energia Elétrica ([2018]), respectivamente.

\subsubsection{5\% da carga nominal}

O segundo arranjo experimental alimentado pelo inversor e conectado à rede, examinou-se com situação de $85 \%$ carga nominal.

Neste caso, foram analisados os harmônicos com uma carga de 2.400VA. Observouse que o DTT de corrente tem o valor de 3,43\% e suas respectivas contribuições harmônicas estão disponíveis no quadro 9.

Quadro 9 - Harmônicos de Corrente, sistema alimentado pelo inversor, com 85\% da carga nominal.

\begin{tabular}{|l|l|l|}
\hline$N^{\circ}$ da ordem & $I_{\text {rms }}(\mathrm{A})$ & \% fundamental \\
\hline 1 & 10,54 & 100 \\
\hline 3 & 0,05 & 0,54 \\
\hline 5 & 0,26 & 2,54 \\
\hline 7 & 0,12 & 1,13 \\
\hline 9 & 0,05 & 0,46 \\
\hline 11 & 0,04 & 0,36 \\
\hline
\end{tabular}

Fonte: Autor (2020). 
A figura 8 representa a organização das informações do quadro 9, com o objetivo de traduzir o comportamento dos harmônicos de corrente de ordem impar em relação à fundamental.

Figura 8 - Harmônicos de corrente, sistema alimentado pelo inversor, 85\% da carga nominal.

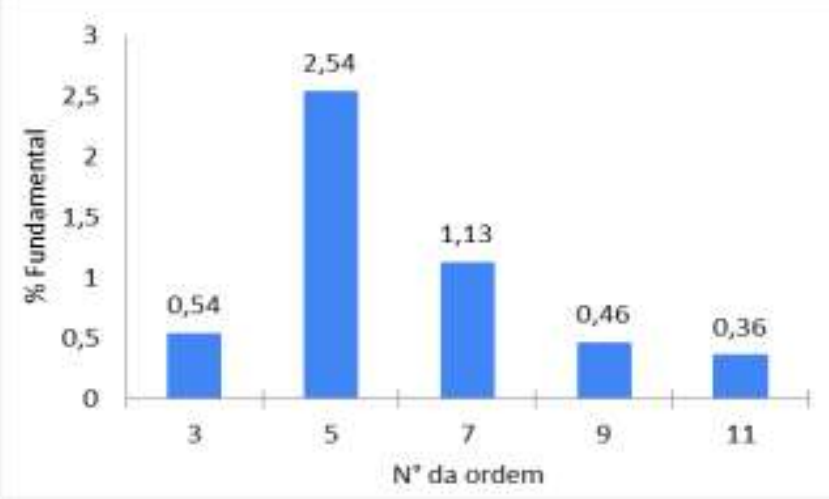

Fonte: Autor (2020).

Analisando os harmônicos de tensão, na situação do sistema alimentado pelo inversor conectado à rede, com $85 \%$ da carga nominal, pode-se averiguar o cenário expresso no quadro 10. O valor numérico do de DTT de tensão, será expresso em 2,66\%.

Quadro 10 - Harmônicos de Tensão, sistema alimentado pelo inversor, com 85\% de carga nominal.

\begin{tabular}{|l|l|l|}
\hline$N^{\circ}$ da ordem & $V_{\text {rms }}(A)$ & \% fundamental \\
\hline 1 & 218,52 & 100 \\
\hline 3 & 1,34 & 0,61 \\
\hline 5 & 5,89 & 2,70 \\
\hline 7 & 1,55 & 0,71 \\
\hline
\end{tabular}

Fonte: Autor (2020).

A figura 9 representa as informações do quadro 10, com a intenção de representar o comportamento dos harmônicos de tensão de ordem impar em relação a fundamental, de maneira gráfica. 
Figura 9 - Harmônicos de tensão, sistema alimentado pelo inversor, 85\% da carga nominal.

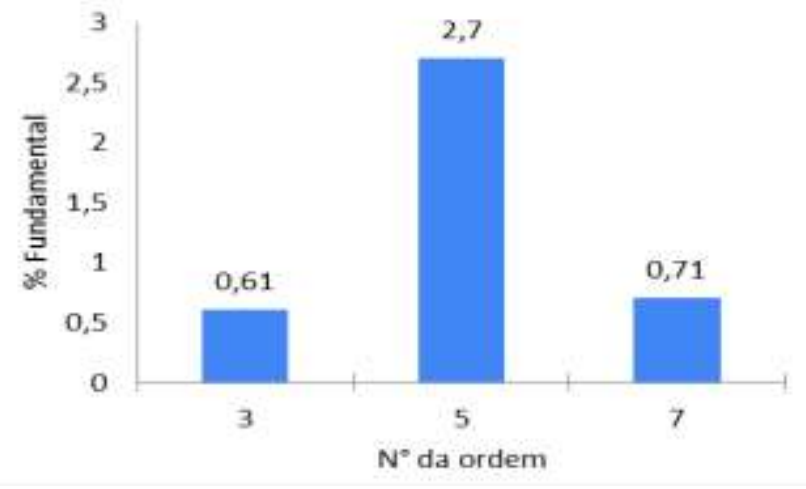

Fonte: Autor (2020).

Os valores do DTT de corrente e tensão estão dentro dos valores normalizados, Institute Of Electrical And Electronics Engineers (1992) e Agência Nacional De Energia Elétrica ([2018]), respectivamente. Contudo, nesta situação de carregamento foi possível observar a participação de ordens harmônicas mais elevadas de corrente, conforme figura 8, quando comparadas com a situação de carregamento anterior (figura 6).

\subsubsection{0\% da carga nominal}

No terceiro arranjo experimental alimentado pelo inversor e conectado à rede examinou-se com situação de $60 \%$ carga nominal.

Nesta problemática, foram analisados os harmônicos com uma carga de 1.900VA. Investigou-se que o DTT de corrente tem o valor de 3,80\% e suas respectivas contribuições harmônicas estão disponíveis no quadro 11.

Quadro 11: Harmônicos de Corrente, sistema alimentado pelo inversor, com 60\% da carga nominal.

\begin{tabular}{|l|l|l|}
\hline$N^{\circ}$ da ordem & $I_{\text {rms }}(\mathrm{A})$ & \% fundamental \\
\hline 1 & 8,65 & 100 \\
\hline 3 & 0,07 & 0,88 \\
\hline 5 & 0,19 & 2,21 \\
\hline 7 & 0,12 & 1,38 \\
\hline 9 & 0,05 & 0,55 \\
\hline 11 & 0,04 & 0,44 \\
\hline 13 & 0,03 & 0,38 \\
\hline
\end{tabular}


A figura 10 equivale à organização dos dados do quadro 11, com o objetivo de traduzir o comportamento dos harmônicos de corrente de ordem impar em relação à fundamental.

Figura 10 - Harmônicos de corrente, sistema alimentado pelo inversor, 60\% da carga nominal.

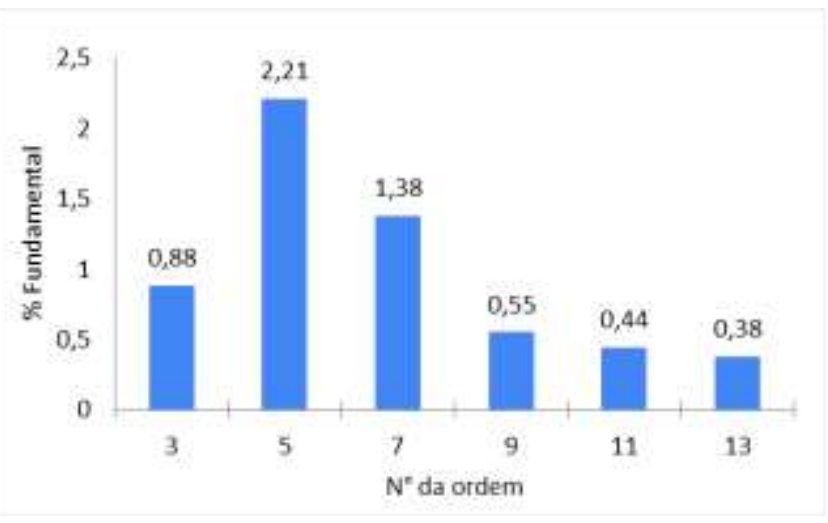

Fonte: Autor (2020).

Explorando os harmônicos de tensão, na situação do sistema alimentado pelo inversor conectado à rede, com $60 \%$ carga nominal, pode-se observar o cenário expresso no quadro 12. É possível verificar numericamente que o valor do DTT de tensão, ficou em $2,62 \%$.

Quadro 12 - Harmônicos de Tensão, sistema alimentado pelo inversor, com 60\% de carga nominal.

\begin{tabular}{|l|l|l|}
\hline$N^{\circ}$ da ordem & $V_{\text {rms }}(A)$ & \% fundamental \\
\hline 1 & 218,57 & 100 \\
\hline 3 & 1,34 & 0,61 \\
\hline 5 & 5,30 & 2,43 \\
\hline 7 & 1,68 & 0,77 \\
\hline
\end{tabular}

Fonte: Autor (2020).

A figura 11 representa as informações do quadro 12, objetivando representar o comportamento dos harmônicos de tensão de ordem impar em relação à fundamental, de maneira gráfica. 
Figura 11 - Harmônicos de tensão, sistema alimentado pelo inversor, 60\% da carga nominal.

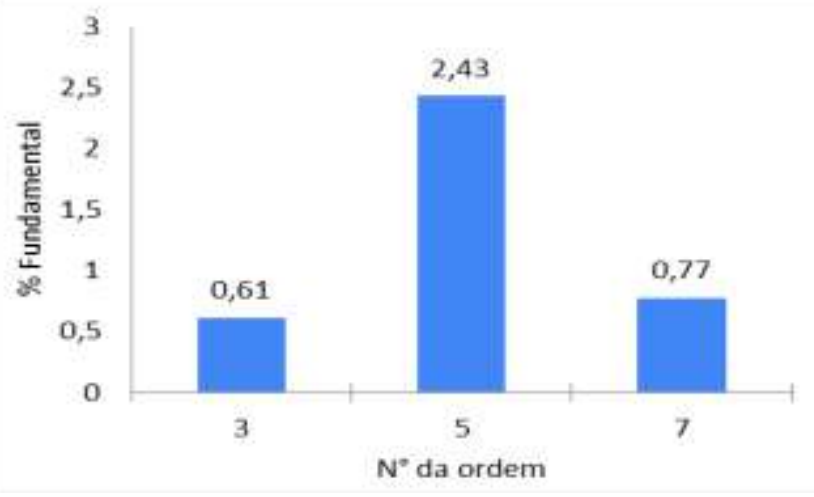

Fonte: Autor (2020).

Os valores do DTT de corrente e tensão estão dentro dos valores normalizados, Institute Of Electrical And Electronics Engineers (1992) e Agência Nacional De Energia Elétrica ([2018]), respectivamente. Contudo, nesta situação de carregamento foi possível observar a participação de ordens harmônicas mais elevadas de corrente, conforme figura 10, quando comparadas com a situação de carregamento (figura 8).

\subsubsection{5\% da carga nominal}

O quarto arranjo experimental alimentado pelo inversor e conectado à rede, examina-se com situação de $45 \%$ carga nominal.

Nesta situação, foram analisados os harmônicos com uma carga de 1.200VA. Verifica-se que o DTT de corrente tem o valor de 5,86\% e suas respectivas contribuições harmônicas estão disponíveis no quadro 13.

Quadro 13 - Harmônicos de Corrente, sistema alimentado pelo inversor, com 45\% da carga nominal.

\begin{tabular}{|l|l|l|}
\hline $\mathrm{N}^{\circ}$ da ordem & $\mathrm{I}_{\mathrm{rms}}(\mathrm{A})$ & \% fundamental \\
\hline 1 & 5,40 & 100 \\
\hline 3 & 0,07 & 1,41 \\
\hline 5 & 0,16 & 3,02 \\
\hline 7 & 0,11 & 2,04 \\
\hline 9 & 0,05 & 0,98 \\
\hline 11 & 0,038 & 0,70 \\
\hline 13 & 0,043 & 0,80 \\
\hline
\end{tabular}




\begin{tabular}{|l|l|l|}
\hline 15 & 0,014 & 0,26 \\
\hline 17 & 0,024 & 0,44 \\
\hline 19 & 0,033 & 0,61 \\
\hline 33 & 0,019 & 0,35 \\
\hline 37 & 0,014 & 0,26 \\
\hline 41 & 0,014 & 0,26 \\
\hline
\end{tabular}

Fonte: Autor (2020).

A figura 12 equivale à organização dos dados do quadro 13, em razão de traduzir o comportamento dos harmônicos de corrente de ordem impar em relação à fundamental.

Figura 12 - Harmônicos de corrente, sistema alimentado pelo inversor, 45\% da carga nominal.

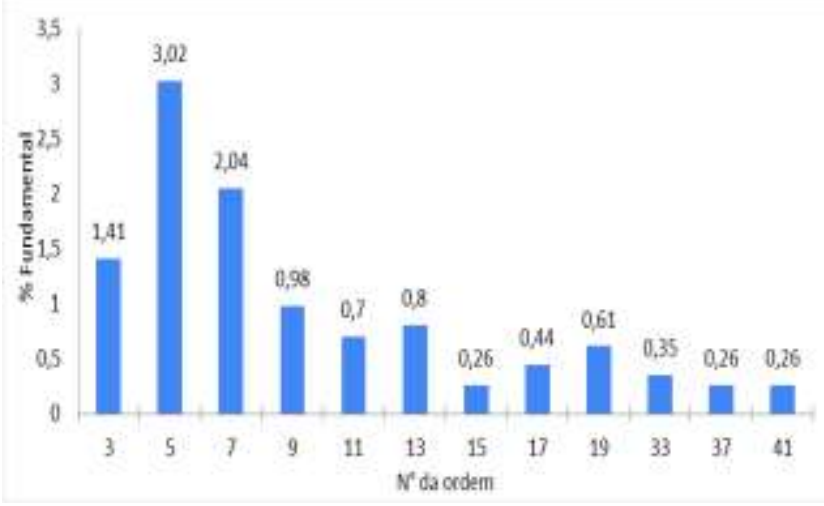

Fonte: Autor (2020).

Avaliando os harmônicos de tensão, na situação do sistema alimentado pelo inversor conectado à rede, com $45 \%$ carga nominal, pode-se analisar o cenário expresso no quadro 14. O valor numérico do de DTT de tensão, será expresso em 2,51\%.

Quadro 14 - Harmônicos de Tensão, sistema alimentado pelo inversor, com 45\% de carga nominal.

\begin{tabular}{|l|l|l|}
\hline $\mathrm{N}^{\circ}$ da ordem & $\mathrm{V}_{\text {rms }}(\mathrm{A})$ & \% fundamental \\
\hline 1 & 218,21 & 100 \\
\hline 3 & 1,32 & 0,61 \\
\hline 5 & 5,04 & 2,31 \\
\hline 7 & 1,71 & 0,79 \\
\hline
\end{tabular}

Fonte: Autor (2020). 
A figura 13 representa as informações do quadro 14, objetivando demonstrar o comportamento dos harmônicos de tensão de ordem impar em relação à fundamental, de maneira gráfica.

Figura 13 - Harmônicos de tensão, sistema alimentado pelo inversor, 45\% da carga nominal.

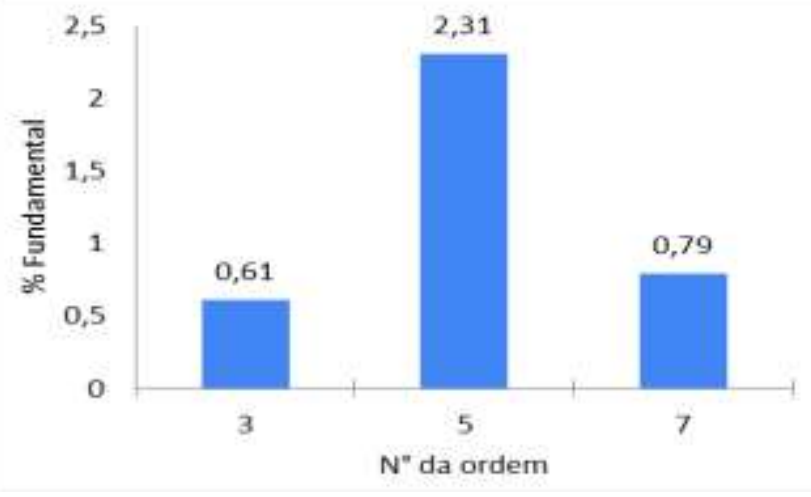

Fonte: Autor (2020).

Os valores do DTT de corrente e tensão estão dentro dos valores normalizados, Institute Of Electrical And Electronics Engineers (1992) e Agência Nacional De Energia Elétrica ([2018]), respectivamente. Contudo, nesta situação de carregamento o foi possível observar o aumento considerável da participação de ordens harmônicas mais elevadas de corrente, conforme figura 12, quando comparadas com a situação de carregamento anterior (figura 10).

\subsubsection{0\% da carga nominal}

O quinto arranjo experimental alimentado pelo inversor e conectado à rede, examinou-se com situação de $30 \%$ carga nominal.

Neste cenário, foram analisados os harmônicos com uma carga de 800VA. Verificouse que o DTT de corrente tem o valor de $8,07 \%$ e suas respectivas contribuições harmônicas estão disponíveis no quadro 15. 
Quadro 15 - Harmônicos de Corrente, sistema alimentado pelo inversor, com 30\% da carga nominal.

\begin{tabular}{|l|l|l|}
\hline $\mathrm{N}^{\circ}$ da ordem & $I_{\text {rms }}(\mathrm{A})$ & \% fundamental \\
\hline 1 & 3,638 & 100 \\
\hline 3 & 0,062 & 1,15 \\
\hline 5 & 0,134 & 2,48 \\
\hline 7 & 0,096 & 1,78 \\
\hline 9 & 0,029 & 0,54 \\
\hline 11 & 0,067 & 1,24 \\
\hline 13 & 0,048 & 0,89 \\
\hline 15 & 0,029 & 0,54 \\
\hline 17 & 0,014 & 0,26 \\
\hline 19 & 0,019 & 0,35 \\
\hline 23 & 0,019 & 0,35 \\
\hline 25 & 0,019 & 0,35 \\
\hline 27 & 0,019 & 0,35 \\
\hline 31 & 0,024 & 0,44 \\
\hline
\end{tabular}

Fonte: Autor (2020).

A figura 14 apresenta a disposição das informações do quadro 15, com o objetivo de traduzir o comportamento dos harmônicos de corrente de ordem impar em relação à fundamental, de maneira gráfica.

Figura 14 - Harmônicos de corrente, sistema alimentado pelo inversor, 30\% da carga nominal.

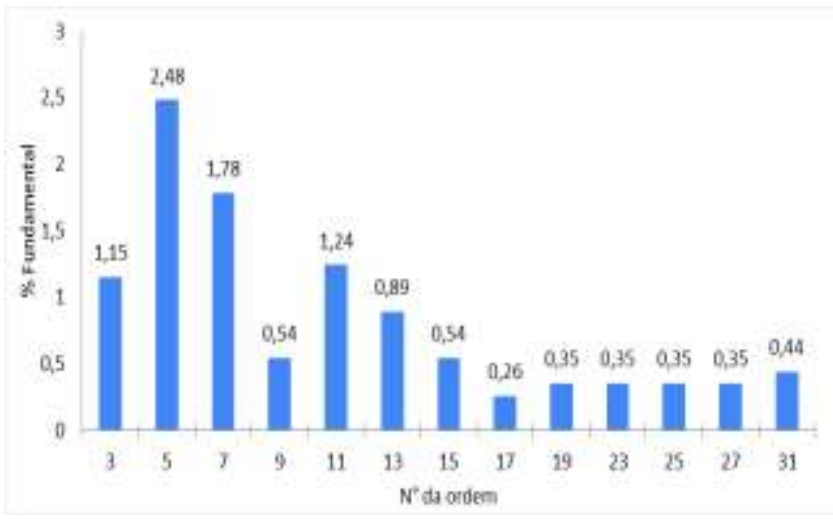

Fonte: Autor (2020).

Avaliando os harmônicos de tensão, na situação do sistema alimentado pelo inversor conectado à rede, com 30\% carga nominal, pode-se analisar o cenário expresso no 
quadro 16. É possível verificar numericamente que o valor do DTT de tensão, ficou em $2,71 \%$.

Quadro 16 - Harmônicos de Tensão, sistema alimentado pelo inversor, com 30\% de carga nominal.

\begin{tabular}{|l|l|l|}
\hline$N^{\circ}$ da ordem & $\mathrm{V}_{\text {rms }}(\mathrm{A})$ & \% fundamental \\
\hline 1 & 218,09 & 100 \\
\hline 3 & 1,37 & 0,63 \\
\hline 5 & 5,48 & 2,52 \\
\hline 7 & 1,74 & 0,80 \\
\hline
\end{tabular}

Fonte: Autor (2020).

A figura 15 representa as informações do quadro 16, com a intenção de expressar o comportamento dos harmônicos de tensão de ordem impar em relação à fundamental, de maneira gráfica.

Figura 15 - Harmônicos de tensão, sistema alimentado pelo inversor, 30\% da carga nominal.

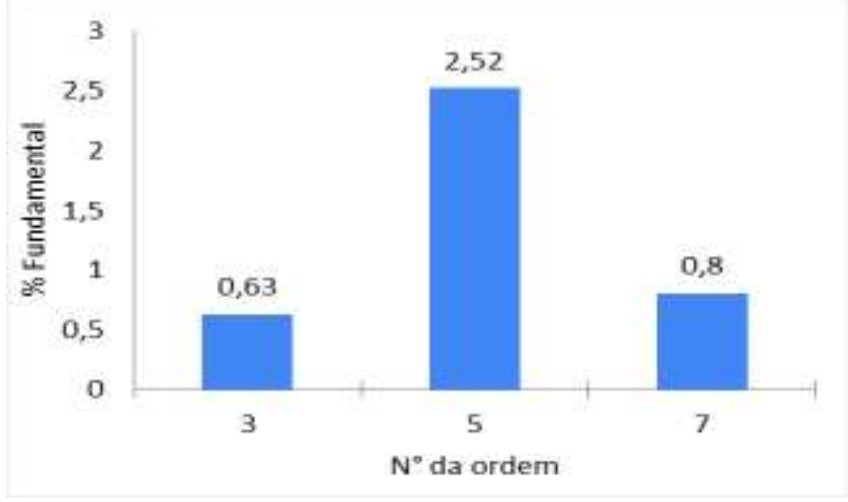

Fonte: Autor (2020).

O valor do DTT de corrente está fora do valor padronizado pelo Institute Of Electrical And Electronics Engineers (1992) e o DTT de tensão estão dentro da norma, segundo a Agência Nacional De Energia Elétrica ([2018]). Contudo, nesta situação de carregamento o foi possível observar a participação de ordens harmônicas mais elevadas de corrente, conforme figura 14, quando comparadas com a situação de carregamento anterior (figura 12). 


\subsubsection{5\% da carga nominal}

O sexto arranjo experimental alimentado pelo inversor e conectado à rede, examinou-se com situação de $15 \%$ carga nominal.

Neste contexto, foram analisados os harmônicos com uma carga de 400VA. Observou-se que o DTT de corrente tem o valor $13,02 \%$ e suas respectivas contribuições harmônicas estão disponíveis no quadro 17.

Quadro 17 - Harmônicos de Corrente, sistema alimentado pelo inversor, com 15\% da carga nominal.

\begin{tabular}{|l|l|l|}
\hline $\mathrm{N}^{\circ}$ da ordem & $\mathrm{I}_{\mathrm{rms}}(\mathrm{A})$ & \% fundamental \\
\hline 1 & 1,993 & 100 \\
\hline 3 & 0,081 & 1,50 \\
\hline 5 & 0,095 & 1,76 \\
\hline 7 & 0,119 & 2,20 \\
\hline 9 & 0,043 & 0,80 \\
\hline 11 & 0,019 & 0,35 \\
\hline 13 & 0,014 & 0,26 \\
\hline 15 & 0,005 & 0,09 \\
\hline 17 & 0,009 & 0,17 \\
\hline 19 & 0,009 & 0,17 \\
\hline 21 & 0,014 & 0,26 \\
\hline 23 & 0,005 & 0,09 \\
\hline 25 & 0,005 & 0,09 \\
\hline 27 & 0,005 & 0,09 \\
\hline 31 & 0,009 & 0,17 \\
\hline 33 & 0,009 & 0,17 \\
\hline 35 & 0,005 & 0,09 \\
\hline 37 & 0,009 & 0,17 \\
\hline
\end{tabular}

Fonte: Autor (2020).

A figura 16 apresenta a disposição das informações do quadro 17, com o objetivo de traduzir o comportamento das harmônicas de corrente de ordem impar em relação a fundamental, de maneira gráfica. 
Figura 16 - Harmônicos de corrente, sistema alimentado pelo inversor, 15\% da carga nominal.

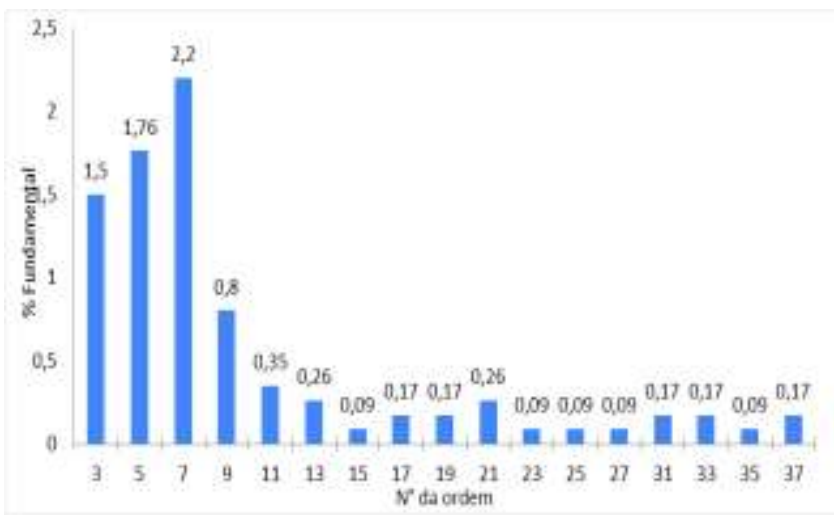

Fonte: Autor (2020).

Avaliando-se os harmônicos de tensão, na situação do sistema alimentado pelo inversor conectado à rede, com $15 \%$ carga nominal, encontra-se cenário expresso no quadro 18. O valor numérico do de DTT de tensão, será expresso em $2,60 \%$.

Quadro 18 - Harmônicos de Tensão, sistema alimentado pelo inversor, com 15\% de carga nominal.

\begin{tabular}{|l|l|l|}
\hline $\mathrm{N}^{\circ}$ da ordem & $\mathrm{V}_{\mathrm{rms}}(\mathrm{A})$ & \% fundamental \\
\hline 1 & 217,35 & 100 \\
\hline 3 & 1,37 & 0,63 \\
\hline 5 & 5,22 & 2,40 \\
\hline 7 & 1,68 & 0,77 \\
\hline
\end{tabular}

Fonte: Autor (2020).

A figura 17 representa as informações do quadro 18, com a intenção de expressar o comportamento dos harmônicos de tensão de ordem impar em relação à fundamental, de maneira gráfica. 
Figura 17 - Harmônicos de tensão, sistema alimentado pelo inversor, 15\% da carga nominal.

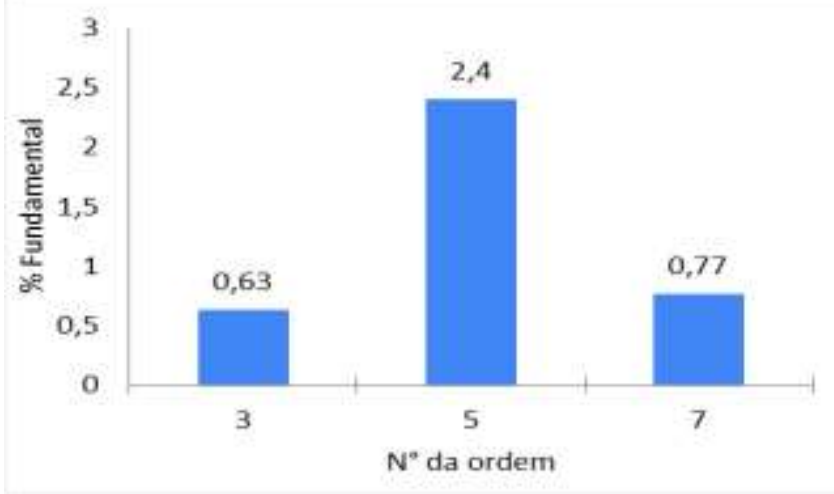

Fonte: Autor (2020).

O valor do DTT de corrente está fora do valor padronizado por Institute Of Electrical And Electronics Engineers (1992) e o DTT de tensão estão dentro da norma, segundo a Agência Nacional De Energia Elétrica ([2018]). Contudo, nesta situação de carregamento o foi possível observar o aumento expressivo da participação de ordens harmônicas mais elevadas de corrente, conforme figura 16, quando comparadas com a situação de carregamento anterior (figura 14).

Após apresentados esses cenários, foi feito um resumo no quadro 19, para centralizar as informações que foram dispostas experimentalmente.

Quadro 19 - Resumo dos Resultados Experimentais.

\begin{tabular}{|l|l|l|}
\hline $\begin{array}{l}\text { Carregamento } \\
(\%)\end{array}$ & THD $_{\mathrm{i}}(\%)$ & THD $_{\mathrm{v}}(\%)$ \\
\hline 100 & 3,48 & 2,85 \\
\hline 85 & 3,43 & 2,66 \\
\hline 60 & 3,80 & 2,62 \\
\hline 45 & 5,86 & 2,51 \\
\hline 30 & 8,07 & 2,71 \\
\hline 15 & 13,02 & 2,60 \\
\hline
\end{tabular}

Fonte: Autor (2020).

A partir do quadro 19, é possível verificar que com a diminuição percentual da carga nominal, ocorre o aumento gradativo no DTT de corrente, medidas experimentalmente.

Notou-se que devido à redução percentual de carga nominal na saída do inversor, as ordens harmônicas superiores de corrente apareciam e nestas situações, ocorreram 
aumentos nas distorções harmônicas de corrente. Considerando que a máxima distorção harmônica total recomendada para corrente é de $8 \%$, segundo o Institute Of Electrical And Electronics Engineers (1992), para as situações de operação com percentuais de carga de $30 \%$ e $15 \%$, o DTT de corrente excedeu o valor limite, chegando a 13,02\% para o carregamento de $15 \%$. O DTT de tensão variou muito pouco, ficando dentro do valor limite de 5\%, em acordo com a Agência Nacional De Energia Elétrica ([2018]).

Em (BLOOMING; CARNOVALE, 2006) há um estudo sobre a aplicação do Institute Of Electrical And Electronics Engineers (1992), por meio de exemplos calculados. O conceito de distorção total de demanda é apresentado nesta pesquisa, no qual este parâmetro depende das correntes de ordem harmônica e da corrente de carga, assim, quanto menor o percentual de carga nominal, menor a corrente de carga e, portanto, maior a distorção total de demanda. A distorção total de demanda é apresentada como sendo diretamente proporcional à distorção harmônica de corrente. Assim, para um percentual de carga menor, a distorção harmônica total de corrente, também aumenta, o que explica os resultados obtidos para a distorção harmônica total de corrente.

\section{CONCLUSÃO}

Os sistemas fotovoltaicos estão em crescimento por todo o território brasileiro. A implantação destes sistemas segue recomendações de instalação das distribuidoras locais, mas recomendações especificas para análise e avaliação dos fenômenos de qualidade de energia ainda merecem maiores contribuições.

Este trabalho apresenta contribuição neste cenário para um pequeno sistema instalado em um campus universitário, com avaliações para modelo de carregamento definido o que não necessariamente é o encontrado nas residências e comércios.

Destaca-se que os estudos apresentados aqui são parte de pesquisas realizadas pelos grupos de estudos na universidade e foco de diferentes abordagens na busca por cenários e possíveis condições de impacto na rede de distribuição de energia elétrica.

O modelo experimental utilizado composto apenas de cargas resistivas possibilita que toda a distorção harmônica total produzida no experimento, tenha existido devido ao conjunto de alimentação e não a estas cargas, ou seja, estas não produzem distorções de corrente ou tensão no sistema analisado. Deve lembrar que as distribuidoras são reguladas 
pela Aneel e que o PRODIST em seu módulo 8 apresenta diversos aspectos com respeito à qualidade de energia distribuído pelas concessionárias, mas não reporta (e não é foco desta regulamentação) o comportamento do cliente conectado à rede de distribuição.

\section{REFERÊNCIAS}

AFONSO, T. A. S. F. et al. Impact Analysis on Power Quality of a Small Distributed Generation. American Journal of Renewable and Sustainable Energy, v. 4, n. 3, p. 56-63, sep. 2018. Disponível em: http://www.aiscience.org/journal/paperlnfo/ajrse? paperld=4136. Acesso em: 02 set. 2020.

AGÊNCIA NACIONAL DE ENERGIA ELÉTRICA. Procedimentos de Distribuição de Energia Elétrica no Sistema Elétrico Nacional - PRODIST. Brasília, DF: ANEEL, [2018]. Disponível em: http://www.aneel.gov.br/documents/656827/14866914/M\%C3\%B3dulo_8-

Revis\%C3\%A3o_10/2f7cb862-e9d7-3295-729a-b619ac6baab9. Acesso em: 5 set. 2020.

AGÊNCIA NACIONAL DE ENERGIA ELÉTRICA. Resolução normativa no 481, de 17 de abril de 2012. Altera a Resolução Normativa no 77, de 18 de agosto de 2004. Brasília, DF: ANEEL, 2012a. Disponível em: http://www2.aneel.gov.br/cedoc/ren2012481.pdf. Acesso em: 5 set. 2020.

AGÊNCIA NACIONAL DE ENERGIA ELÉTRICA. Resolução normativa no 482, de 17 de abril de 2012. Estabelece as condições gerais para o acesso de microgeração e minigeração distribuída aos sistemas de distribuição de energia elétrica, o sistema de compensação de energia elétrica, e dá outras providências. Brasília, DF: ANEEL, 2012b. Disponível em: http://www2.aneel.gov.br/cedoc/ren2012482.pdf. Acesso em: 5 set. 2020.

ALINE. O que é um inversor fotovoltaico?. SolarOn, [S. I.], 2018. Disponível em: http://solaron.eco.br/o-que-e-um-inversor-fotovoltaico/. Acesso em: 1 abr. 2020.

BLOOMING, T. M.; CARNOVALE, D. J. Application of IEEE STD 519-1992 harmonic limits. In: CONFERENCE RECORD OF 2006 ANNUAL PULP AND PAPER INDUSTRY TECHNICAL CONFERENCE, 2006, 2006, Appleton, WI. Anais [...]. IEEE, 2006. p. 1-9.

BOUCHAKOUR, S. et al. Investigation of the voltage quality at PCC of grid connected PV system. Energy Procedia, [S. I.], v. 141, p. 66-70, 2017.

CAMPOS, R. A.; NASCIMENTO, L. R.; RUTHER, R. The complementary nature between wind and photovoltaic generation in Brazil and the role of energy storage in utility-scale hybrid power plants. Energy Conversion and Management, v. 221, oct. 2020. Disponível em: https://www.sciencedirect.com/science/article/abs/pii/S0196890420307044. Acesso em: 1 abr. 2020.

GOFFERJÉ, M. J. Estudo da correção do fator de potência em pontos de conexão entre as redes básica e de distribuição. 2019. 84 p. Trabalho de Conclusão de Curso (Bacharelado em 
Engenharia Elétrica) - Universidade Federal de Santa Catarina, Florianópolis, 2019. Disponível em: https://repositorio.ufsc.br/handle/123456789/195775. Acesso em: 10 jan. 2020.

INSTITUTE OF ELECTRICAL AND ELECTRONICS ENGINEERS. IEEE Std 519: IEEE Recommended Practices and Requirements for Harmonic Control in Electrical Power Systems. [Nova Jersey]: IEEE, 1992.

INSTITUTE OF ELECTRICAL AND ELECTRONICS ENGINEERS. IEEE Std 1547: IEEE Standard for Interconnecting Distributed Resources with Electric Power Systems. [Nova Jersey]: IEEE, 2003.

JURINIC, F. Estudo para melhoria na performance e eficiência de placas fotovoltaicas: através de um sistema combinado de inclinação e resfriamento. 2020. 96 p. Dissertação (Mestrado em Ambiente e Tecnologias Sustentáveis) - Universidade Federal da Fronteira Sul, Rio Grande do Sul, 2020. Disponível em: https://rd.uffs.edu.br/handle/prefix/3612. Acesso em: 10 jan. 2020.

LEITE, M. C. C. et al. Harmonic analysis of a photovoltaic systems connected to low voltage grid. IEEE Latin America Transactions, [S. I.], v. 16, n. 1, p. 112-117, jan. 2018. Disponível em:

https://www.researchgate.net/publication/323197814_Harmonic_Analysis_of_a_Photovolt aic_Systems_Connected_to_Low_Voltage_Grid. Acesso em: 10 jan. 2020.

MAGALHÃES, I. B. et al. Site suitability for photovoltaic energy expansion: a brazilian's high demand states study case. Remote Sensing Applications Society and Environment, [S. I.], v. 19, jun. 2020.

EMPRESA DE PESQUISA ENERGÉTICA (BRASIL). Balanço energético nacional 2015: ano base 2014. Rio de Janeiro: EPE, 2015. Disponível em: https://www.epe.gov.br/sitespt/publicacoes-dados-abertos/publicacoes/PublicacoesArquivos/publicacao-127/topico97/Relat\%C3\%B3rio\%20Final\%202015.pdf. Acesso em: 1 mai. 2020.

EMPRESA DE PESQUISA ENERGÉTICA (BRASIL). Balanço energético nacional 2018: ano base 2017. Rio de Janeiro: EPE, 2018. Disponível em: https://www.epe.gov.br/sitespt/publicacoes-dados-abertos/publicacoes/PublicacoesArquivos/publicacao-303/topico419/BEN2018_Int.pdf. Acesso em: 1 mai. 2020.

PEREIRA, E. B. et al (org.) Atlas brasileiro de energia solar. São José dos Campos: INPE, 2006.

RIBEIRO JR., J.F. Estudo de viabilidade técnica para correção de fator de potência em redes de distribuição rurais monofásicas. 2016. 90 p. Trabalho de conclusão de curso (Bacharelado em Engenharia Elétrica) - Instituto tecnológico de Caratinga, Caratinga, Minas Gerais, 2015. Disponível em: http://dspace.doctum.edu.br/bitstream/123456789/534/2/TCC\%20FRANCISCO\%20PUBLICA DO.pdf. Acesso em: 10 jan. 2020. 
SILVA, B. S. et al. Power quality (PQ) analysis in a power feeder that supply different social class consumers. International Journal of Emerging Electric Power Systems, [S. I.], v. 21, n. $2,2020$.

SOUZA, D. C. R. et al. Analysis of Payback Time in Photovoltaic Systems: Case Study with Two Projects. International Journal of Environmental Planning and Management, [S. I.], v. 5, n. 3, p. 51-58, sep. 2019. Disponível em: http://www.aiscience.org/journal/paperlnfo/ijepm?paperld=4671. Acesso em: 29 mar. 2020.

SOUZA, G.; MOSQUEIRA, R. A.; FORTES, M. Z. Viabilidade Técnico-financeira de Projetos de Geração Fotovoltaica: Análise de Perdas. Revista SODEBRAS, [S. I.], v. 13, n. 153, p. 101-107, set. 2018. Disponível em: http://www.sodebras.com.br/edicoes/N153.pdf. Acesso em: 15 jan. 2020.

VIDAURRE, R. M.; FORTES, M. Z. The interaction of submarine cables and the power quality of an oil rig's electrical system. Electrical Engineering, [S. I.], v. 102, p. 1521-1530, 2020. 